\title{
Mitochondrial pyruvate carrier modulates the epithelial-mesenchymal transition in cholangiocarcinoma
}

\author{
TOMOFUMI OHASHI ${ }^{1-3}$, HIDETOSHI EGUCHI $^{1^{*}}$, KOICHI KAWAMOTO $^{1,2^{*}}$, MASAMITSU KONNO $^{2,3^{*}}$, \\ AYUMU ASAI ${ }^{2,3}$, HUGH COLVIN ${ }^{1-3}$, YUJI UEDA ${ }^{1}$, HIROFUMI TAKAOKA ${ }^{4}$, YOSHIFUMI IWAGAMI ${ }^{1}$, \\ DAISAKU YAMADA ${ }^{1}$, TADAFUMI ASAOKA ${ }^{1}$, TAKEHIRO NODA ${ }^{1}$, HIROSHI WADA $^{1}$, KUNIHITO GOTOH ${ }^{1}$, \\ SHOGO KOBAYASHI ${ }^{1}$, JUN KOSEKI ${ }^{3}$, TAROH SATOH ${ }^{2}$, KAZUHIKO OGAWA $^{4}$, \\ YUICHIRO DOKI ${ }^{1}$, MASAKI MORI $^{1}$ and HIDESHI ISHII ${ }^{2,3}$
}

Departments of ${ }^{1}$ Gastroenterological Surgery, ${ }^{2}$ Frontier Science for Cancer and Chemotherapy, ${ }^{3}$ Medical Data Science and ${ }^{4}$ Radiation Oncology, Graduate School of Medicine, Osaka University, Suita, Osaka 565-0871, Japan

Received August 29, 2017; Accepted November 17, 2017

DOI: 10.3892/or.2017.6172

\begin{abstract}
Intrahepatic cholangiocarcinoma (ICC) is known to have a high malignant potential. Because of its high recurrence rate, ICC has a poor prognosis even after complete tumor resection. Compared with normal differentiated cells, cancer cells have an altered metabolism for supporting their survival in severe conditions. Cancer cells acquire additional malignant potential as a result of this metabolic alteration. Thus, the molecules known to be involved in cancer metabolism, could be novel therapeutic targets. The mitochondrial pyruvate carrier (MPC) is a recently discovered pyruvate transporter, which is located in the mitochondrial inner membrane. Although MPC is composed of two subunits, it has been reported that the MPC1 subunit is specifically associated with poor prognosis in several cancers, including colorectal and prostate cancer. However, only a few studies have assessed the clinical significance of MPC1 and the molecular mechanisms underlying its influence on cancer progression are not well understood. This study aimed to clarify the function of MPC1 that affects the malignant potential of ICC. The expression of MPC1 in ICC clinical specimens was determined by immunohistochemistry. In addition, the correlations between MPC1 expression
\end{abstract}

Correspondence to: Professor Hideshi Ishii, Department of Frontier Science for Cancer and Chemotherapy, Graduate School of Medicine, Osaka University, 2-2 Yamadaoka, Suita, Osaka 565-0871, Japan

E-mail: hishii@gesurg.med.osaka-u.ac.jp

Professor Masaki Mori, Department of Gastroenterological Surgery, Graduate School of Medicine, Osaka University, 2-2 Yamadaoka, Suita, Osaka 565-0871, Japan

E-mail: mmori@gesurg.med.osaka-u.ac.jp

*Contributed equally

Key words: cholangiocarcinoma, mitochondria, MPC, EMT, cancer metabolism and the survival rate, as well as various clinicopathological parameters were assessed. Low MPC1 expression correlated with poor ICC prognosis and was correlated with tumor invasion and distant metastasis. Both these phenomena are closely associated with the epithelial-mesenchymal transition (EMT). Therefore, we investigated the impact of altering the MPC1 gene expression on the malignant potential of cancer cells using biliary tract cancer cell lines in vitro. The expression of MPC1 was downregulated in the cells induced to undergo EMT following treatment with TGF- $\beta$. Furthermore, the inhibition of MPC1 expression induced EMT in cancer cells, and the overexpression of MPC1 suppressed the migration of tumor cells. These results indicated that MPC1 could be a novel therapeutic target in some cancers.

\section{Introduction}

Intrahepatic cholangiocarcinoma (ICC) is the second most common primary liver malignancy and ICC patients often have a poor prognosis. The currently available chemotherapeutic agents have limited efficacy for ICC patients, thus complete resection is the only therapeutic treatment possible at present (1-3). Unfortunately, the recurrence rate of ICC after complete resection, is comparatively high, due to its high malignant potential (4).

There is a growing interest in cancer metabolism with many studies indicating that metabolic alterations contribute to malignant potential (5-7). Cancer cells alter their metabolism, which promotes their survival, in response to changes in the tumor microenvironment. Normal cells depend on mitochondrial oxidative phosphorylation for adenosine triphosphate (ATP) production, while cancer cells depend on glycolysis, under both aerobic and anaerobic conditions. This metabolic phenomenon is known as the Warburg effect (also known as aerobic glycolysis) and is recognized as an adaptation to the tumor environment $(8,9)$. Multiple molecules and steps are involved in this metabolic alteration, for example, lactate dehydrogenase A and pyruvate kinase M2 (PKM2) play pivotal roles in aerobic glycolysis by limiting the production of pyruvate, which is essential for mitochondrial oxidative 
phosphorylation $(10,11)$. Another mechanism of aerobic glycolysis, although not mutually exclusive, is the inhibition of pyruvate import into the mitochondrial matrix, resulting in decreased pyruvate oxidation associated with the increase of lactate production.

Recently, the mitochondrial pyruvate carrier (MPC) was identified as a complex of two subunits, MPC1 and MPC2, located in the mitochondrial inner membrane $(12,13)$. Pyruvate is transported from the cytoplasm into the mitochondria via the MPC and is then used for the production of ATP in the tricarboxylic acid cycle in the mitochondrial matrix. The MPC functions at the pyruvate branch point, therefore, alterations in the expression or activity of the MPC affect metabolism in both normal and cancer cells $(14,15)$. The downregulation of MPC1 has been observed in solid cancers, such as colon and prostate cancer, and is reported to be correlated with a poor prognosis (16-18). However, there are no studies describing the relationship between the expression of MPC1 and the prognosis in ICC. Additionally, the contribution of MPC1 to the malignant potential of cancer cells is unclear. This study aimed to assess both the significance of the MPC1 expression in ICC and the role of MPC1 in the malignant potential of cancer cells.

\section{Materials and methods}

Patients and specimens. Sixty-four patients with ICC who underwent curative surgery at Osaka University Hospital from March 1998 to November 2014 were included in this study. Patients who had received preoperative chemotherapy were excluded. Table I summarizes the characteristics of the patients under the ethical approval of the study as well as the written informed consents of the patients (by M.M. and H.I.; protocol nos. 09412; 2703-4; 24-122-011).

Immunohistochemistry (IHC). We performed IHC for MPC1 using the following method: formalin-fixed, paraffin-embedded sections $(4 \mu \mathrm{m})$ were deparaffinized in xylene, boiled for antigen retrieval and incubated overnight at $4^{\circ} \mathrm{C}$ with an anti-MPC1 antibody (HPA045119, 1:500 dilution; Sigma-Aldrich, St. Louis, MO, USA). The sections were first stained with the avidin-biotin complex (Vector Laboratory, Burlingame, CA, USA) and diaminobenzidine and then counterstained with hematoxylin. Five fields at x100 magnification were randomly selected from each sample for IHC analysis. Based on the intensity of the cytoplasmic staining for MPC1, the samples were scored and assigned to one of four categories: 0 , no staining; 1 , weak staining; 2 , moderate staining; and 3 , strong staining.

Cell lines, cultures and drugs. We used the human biliary tract cancer (BTC) cell lines TFK-1 and CCLP-1. The TFK-1 cell line was obtained from the Riken BioResource Center (Tsukuba, Ibaragi, Japan) and the CCLP-1 cell line was generously provided by Dr Gregory J. Gores of the Mayo Clinic (Rochester, MN, USA). The cells were cultured in Dulbecco's modified Eagle's medium (DMEM D6046; Sigma-Aldrich) supplemented with $10 \%$ fetal bovine serum (FBS) and $1 \%$ penicillin-streptomycin (Invitrogen Life Technologies, Carlsbad, CA, USA) and then incubated at $37^{\circ} \mathrm{C}$ in a humidified incubator containing $5 \% \mathrm{CO}_{2}$. The cell lines were treated either with $5 \mathrm{ng} / \mathrm{ml}$ recombinant human transforming growth factor- $\beta 1$ (TGF- $\beta 1$ ) (PeproTech, Rocky Hill, NJ, USA) for induction of the EMT, or with phosphate-buffered saline (PBS) as a negative control.

Transfection. MPC1 small interfering (siRNA) oligonucleotide (siMPC1) and a scrambled oligonucleotide were purchased from Applied Biosystems (Thermo Fisher Scientific, Inc., Foster City, CA, USA). Both the siMPC1 oligonucleotide and the scrambled oligonucleotide were transfected using Lipofectamine 3000 reagent (Invitrogen Life Technologies) according to the manufacturer's protocol.

Plasmids. The CCLP-1 cells were transfected with the pcDNA3-MPC1 expression vector, generated in our laboratory, or with the pcDNA3 empty vector as a negative control (Invitrogen Life Technologies). The vectors were transfected using Lipofectamine 3000 and P3000 reagents (Invitrogen Life Technologies) according to the manufacturer's protocol.

Quantitative real-time PCR. Total RNA was extracted from the cells using TRIzol reagent (Invitrogen Life Technologies). Complementary DNA was synthesized using ReverTra Ace (Toyobo, Osaka, Japan) and real-time PCR was conducted with the Thunderbird SYBR qPCR mix (Toyobo). The primers were as follows: $\beta$-actin forward, 5'-GATGAGATTGGCATG GCTTT-3' and reverse, 5'-CACCTTCACCGTTCCAGTTT-3'; MPC1 forward, 5'-GTGCGGAAAGCGGCGGACTA-3' and reverse, 5'-GGCAGCAATGGGAAGACCCCA-3'; and E-cadherin forward, 5'-ACACCATCCTCAGCCAAGA-3' and reverse, 5'-CGTAGGGAAACTCTCTCGGT-3'.

Western blot analysis. Total protein was extracted from the BTC cell lines with RIPA buffer (Thermo Fisher Scientific, Inc., Rockford, IL, USA) and aliquots of protein were electrophoresed on SDS-PAGE Tris-HCl gels (Bio-Rad Laboratories, Hercules, CA, USA). The separated proteins were transferred to Immuno-Blot PVDF membranes (Bio-Rad Laboratories) using a wet transfer system. The membranes were then incubated overnight at $4^{\circ} \mathrm{C}$ with the MPC1 antibody (HPA045119, 1:250 dilution; Sigma-Aldrich), E-cadherin antibody (sc-7870, 1:500 dilution; Santa Cruz Biotechnology, Dallas, TX, USA) or ACTB antibody (A2066, 1:2,000 dilution; Sigma-Aldrich) followed by an $1 \mathrm{~h}$ incubation with HRP-linked anti-rabbit IgG (1:100,000 dilution; GE Healthcare Biosciences, Piscataway, NJ, USA) at room temperature. The antigen-antibody complex was detected with an ECL Prime Western Blotting Detection kit (GE Healthcare Biosciences).

Reactive oxygen species (ROS). ROS detection was performed using a CellROX Deep Red Flow Cytometry Assay kit (C10491; Thermo Fisher Scientific) according to the manufacturer's protocol. Briefly, the TFK-1 cells were transfected with siMPC1 or scrambled oligonucleotide and cultured for $24 \mathrm{~h}$ and then the CellROX Reagent was added at a final concentration of $5 \mu \mathrm{M}$. After incubation for $30 \mathrm{~min}$ at $37^{\circ} \mathrm{C}$, the cells were fixed with $3.7 \%$ formaldehyde for $15 \mathrm{~min}$ and analyzed using BD FACSAria II (Becton, Dickinson and Company, Franklin Lakes, NJ, USA). 
Table I. Clinicopathological characteristics of the 64 patients in the study.

\begin{tabular}{lc}
\hline Variables & Data $(\mathrm{n}=64)$ \\
\hline Age (years) & $62.0 \pm 11.7$ \\
Sex (male/female) & $40 / 24$ \\
Hepatitis (negative/HBV/HCV/HBV+HCV) & $46 / 11 / 6 / 1$ \\
$\mathrm{CEA}(\leq 5 />5 \mathrm{ng} / \mathrm{ml})$ & $53 / 11$ \\
CA19-9 $(\leq 37 />37 \mathrm{U} / \mathrm{ml})$ & $38 / 26$ \\
Operation time (min) & $492 \pm 222$ \\
Blood loss $(\mathrm{ml})$ & $1.371 \pm 1.261$ \\
pT $(1 / 2 / 3 / 4)$ & $1 / 25 / 26 / 12$ \\
Tumor size $(\mathrm{mm})$ & $49.3 \pm 32.8$ \\
Tumor no. (single/multiple) & $47 / 17$ \\
Vascular invasion (no/yes) & $39 / 25$ \\
pN $(0 / 1)$ & $42 / 22$ \\
UICC pStage $(1 / 2 / 3 / 4 \mathrm{a} / 4 \mathrm{~b})$ & $21 / 12 / 1 / 30 / 0$ \\
Histological type (well/moderate/poor/other) & $4 / 39 / 11 / 10$ \\
\hline
\end{tabular}

CEA, carcinoembryonic antigen; CA19-9, carbohydrate antigen 19-9; UICC, Union for International Cancer Control.

Proliferation assay. The cell viability was assessed with a proliferation assay using the Cell Counting Kit-8 (CCK-8; Dojindo Laboratories, Kumamoto, Japan) according to the manufacturer's protocol.

Wound healing assay. The migratory ability was assessed with a wound healing assay as previously described (19). Briefly, the cells were cultured in serum-starved DMEM in a 6-well plate until 90-100\% confluency was reached, monolayers were then scratched with a $200 \mu \mathrm{l}$ pipette tip and after $24 \mathrm{~h}$ the infiltration of the cells into the wounded (scratched) area was quantified.

Statistical analysis. Data are expressed as the mean \pm standard deviation (SD). Statistical analysis was performed using JMP Pro version 10 statistical software (SAS Institute, Cary, NC, USA). Statistically significant differences were determined by the Student's t-test, $\chi^{2}$ or Fisher's exact tests. Recurrence-free survival (RFS) and overall survival (OS) were analyzed by the Kaplan-Meier method and statistical significance was evaluated by the log-rank test. P-values of $<0.05$ were considered indicative of statistical significance.

\section{Results}

Low MPC1 expression in ICC specimens is correlated with poor prognosis. We investigated the expression of MPC1 in resected specimens of ICC by IHC. The characteristics of the 64 patients in this study are summarized in Table I. As depicted in Fig. 1A-a-d, the IHC specimens were divided into four categories according to the staining intensity. After the median values of the staining intensities in each specimen were calculated, the patients were divided into two groups, defined as MPC1 low-expression (MPC1 $\left.1^{\text {low }}\right)$ and
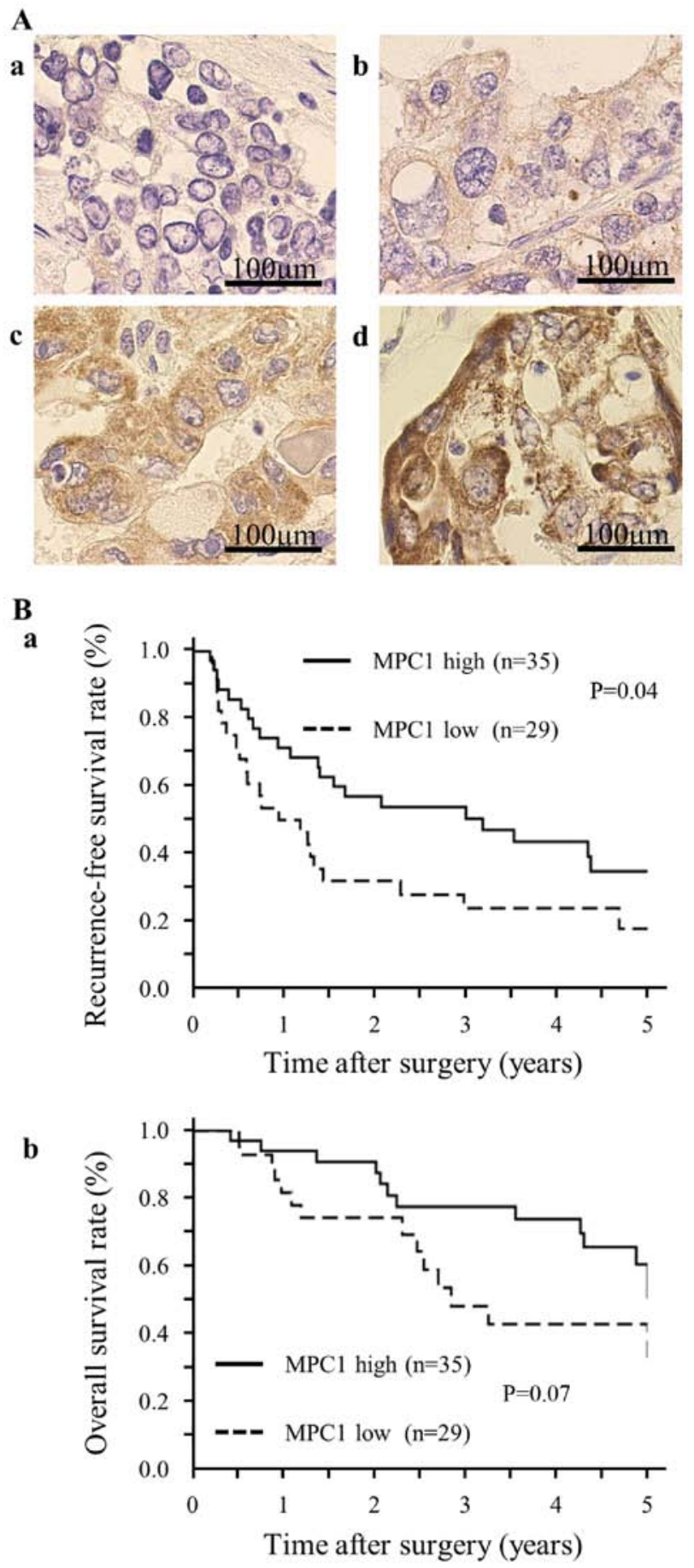

Figure 1. Low expression of MPC1 correlates with a poor prognosis in ICC. (A) Representative IHC images of MPC1 in ICC (scale bar, $100 \mu \mathrm{m}$ ); a) IHC score 0 (no staining); b) IHC score 1 (weakly positive); c) IHC score 2 (moderately positive); d) IHC score 3 (strongly positive). (B) Prognostic analysis of IHC results: a) Kaplan-Meier survival curves of recurrence-free survival; b) Kaplan-Meier survival curves of overall survival. ICC, intrahepatic cholangiocarcinoma IHC, immunohistochemistry.

MPC1 high-expression (MPC $1^{\text {high }}$ ). The Kaplan-Meier curves demonstrated that RFS in the MPC1 ${ }^{\text {low }}$ group was significantly lower than that in the MPC1 ${ }^{\text {high }}$ group (Fig. 1B-a). Although the difference was not statistically significant, we confirmed the same trend for OS (Fig. 1B-b). The clinicopathological analysis revealed that low MPC1 expression correlated with CA19-9 levels and vascular invasion (Table II). We also confirmed 
Table II. Correlation between the MPC1 expression and the clinicopathological variables in patients with ICC.

\begin{tabular}{|c|c|c|c|c|}
\hline & $\begin{array}{c}\text { Total } \\
(\mathrm{n}=64) \\
(\%)\end{array}$ & $\begin{array}{l}\mathrm{MPC}^{\text {low }} \\
(\mathrm{n}=29) \\
(\%)\end{array}$ & $\begin{array}{l}\mathrm{MPC}^{\text {high }} \\
(\mathrm{n}=35) \\
(\%)\end{array}$ & P-value \\
\hline Age (years) & & & & 1.00 \\
\hline$<65$ & $32(50)$ & $15(52)$ & $17(49)$ & \\
\hline$\geq 65$ & $32(50)$ & $14(48)$ & $18(51)$ & \\
\hline Sex & & & & 1.00 \\
\hline Male & $40(63)$ & $18(62)$ & $22(63)$ & \\
\hline Female & $24(37)$ & $11(38)$ & $13(37)$ & \\
\hline Hepatitis & & & & 0.10 \\
\hline No & $46(72)$ & $24(83)$ & $22(63)$ & \\
\hline Yes & $18(28)$ & $5(17)$ & $13(37)$ & \\
\hline CEA (ng/ml) & & & & 0.53 \\
\hline$<5$ & $53(83)$ & $23(79)$ & $30(86)$ & \\
\hline$\geq 5$ & $11(17)$ & $6(21)$ & $5(14)$ & \\
\hline CA19-9 (U/ml) & & & & 0.04 \\
\hline$<37$ & $38(59)$ & $13(45)$ & $25(71)$ & \\
\hline$\geq 37$ & $26(41)$ & $16(55)$ & $10(29)$ & \\
\hline $\mathrm{pT}$ & & & & 0.45 \\
\hline $1+2$ & $26(41)$ & $10(34)$ & $16(46)$ & \\
\hline $3+4$ & $38(59)$ & $19(66)$ & $19(54)$ & \\
\hline Tumor size (mm) & & & & 0.28 \\
\hline$<50$ & $43(67)$ & $17(59)$ & $26(74)$ & \\
\hline$\geq 50$ & $21(33)$ & $12(41)$ & $9(26)$ & \\
\hline Tumor no. & & & & 0.16 \\
\hline Single & $47(73)$ & $24(83)$ & $23(66)$ & \\
\hline Multiple & $17(27)$ & $5(17)$ & $12(34)$ & \\
\hline Vascular invasion & & & & 0.02 \\
\hline No & $39(61)$ & $13(45)$ & $26(74)$ & \\
\hline Yes & $25(39)$ & $16(55)$ & $9(26)$ & \\
\hline $\mathrm{pN}$ & & & & 1.00 \\
\hline 0 & $42(66)$ & $19(66)$ & $23(66)$ & \\
\hline 1 & $22(34)$ & $10(34)$ & $12(34)$ & \\
\hline UICC pStage & & & & 0.80 \\
\hline $1+2$ & $33(52)$ & $14(48)$ & $19(54)$ & \\
\hline $3+4 a$ & $31(48)$ & $15(52)$ & $16(46)$ & \\
\hline Histological type & & & & 0.59 \\
\hline tub1 + tub2 & $43(67)$ & $18(62)$ & $25(71)$ & \\
\hline por + others & $21(33)$ & $11(38)$ & $10(29)$ & \\
\hline $\begin{array}{l}\text { Distant metastasis } \\
\text { (During follow-up } \\
\text { time) }\end{array}$ & & & & 0.04 \\
\hline No & $42(66)$ & $16(55)$ & $26(79)$ & \\
\hline Yes & $20(34)$ & $13(45)$ & $7(21)$ & \\
\hline
\end{tabular}

MPC, mitochondrial pyruvate carrier; ICC, intrahepatic cholangiocarcinoma; CEA, carcinoembryonic antigen; CA19-9, carbohydrate antigen 19-9; UICC, Union for International Cancer Control; tub1, well differentiated; tub2, moderately differentiated: por, poorly differentiated. that among the patients who relapsed during the follow-up period, the percentage of distant metastasis (including lung and bone metastasis and peritoneal dissemination) was significantly higher in the $\mathrm{MPCl}^{\text {low }}$ group than in the MPC $1^{\text {high }}$ group (Table II). Since the most well-known phenomenon involved in invasion and metastasis of cancer cells is EMT, these findings support the hypothesis that the function of MPC1 is strongly related to EMT.

MPCl expression is downregulated in cells induced to undergo EMT by TGF- $\beta$. We used human BTC cell lines (CCLP-1 and TFK-1) to investigate the role of MPC1 in EMT. We confirmed the EMT state in each cell line. CCLP-1 cells formed spindle-like shapes and expressed significantly low levels of E-cadherin (in both mRNA and protein). In contrast, TFK-1 cells formed valvate-like shapes and expressed a high level of E-cadherin. In addition, the expression of MPC1 in the TFK-1 cells was significantly lower than that in the CCLP-1 cells (Fig. 2). These results indicated that the relationship between the expression of E-cadherin and MPC1 is similar in both cell lines and that the MPC1 expression changes when cancer cells progress from the pre-EMT to the post-EMT state. To further investigate this phenomenon, based on the observed characteristics of the cell lines, we defined the TFK-1 cells as pre-EMT and the CCLP- 1 cells as post-EMT. TGF- $\beta 1$ is known to play an important role in EMT induction and has been previously used as an EMT inducer in vivo (20). To confirm the change of the MPC1 expression before and after EMT, we used human recombinant TGF- $\beta 1$ to induce EMT in the TFK- 1 cells. Treatment with $5 \mathrm{ng} / \mathrm{ml}$ TGF- $\beta 1$ caused the TFK-1 cells to undergo a morphological change from a valvate-like shape to a spindle-like shape in a time-dependent manner (Fig. 3A). In addition, the TGF- $\beta 1$ treatment significantly decreased the expression of E-cadherin (Fig. 3B and C). These results demonstrated the change in the TFK-1 cells from a pre-EMT state to a post-EMT state following the TGF- $\beta$ treatment. The TKF-1 cells had a lower MPC1 expression in the post-EMT state than in the pre-EMT state indicating that the expression of MPC1 decreases after progression through the EMT. In addition, it is possible that MPC1 may be directly or indirectly affected by the TGF- $\beta 1$ signaling.

Knockdown of MPC1 leads to induction of EMT. To confirm the role of MPC1 in EMT, we transfected siMPC1 into TFK-1 cells. Surprisingly, the MPC1 knockdown by siRNA transfection caused a morphological change from a valvate-like shape to a spindle-like shape in the TFK-1 cells (Fig. 4A). Additionally, E-cadherin expression was significantly decreased in response to MPC1 knockdown (Fig. 4B and C). These results were consistent with those observed in TFK-1 cells after TGF- $\beta$ treatment and indicated that MPC1 functions as a key modulator of EMT induction in the same way as TGF- $\beta$. We also confirmed that ROS levels significantly decreased in response to MPC1 knockdown (Fig. 4D).

Overexpression of MPCl suppresses tumor cell migration and growth. We then investigated whether the overexpression of the MPC1 gene in the CCLP-1 cells caused phenotypic alterations (Fig. 5A). We confirmed that the cell viability was not affected by the overexpression of the MPC1 gene using 
A

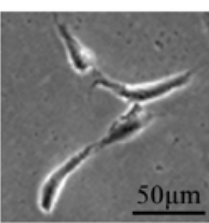

CCLP-1

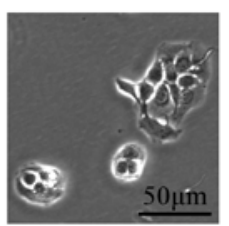

TFK-1
B

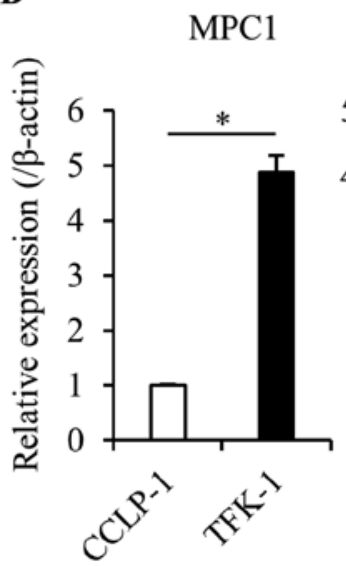

E-cadherin

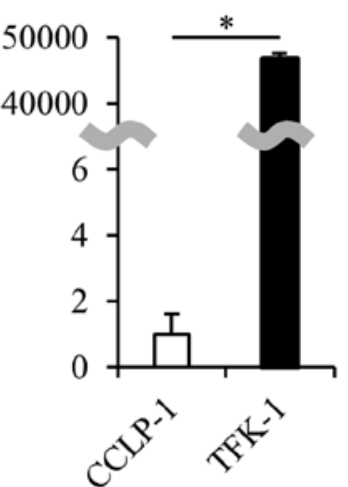

C

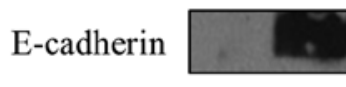

MPC1

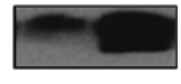

$\beta$-actin
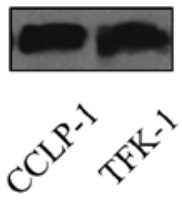

Figure 2. BTC cell line characteristics. (A) Cellular morphology of TFK-1 and CCLP-1 cells (scale bar, $50 \mu \mathrm{m}$ ). (B) Real-time qRT-PCR and (C) western blot results of MPC1 and E-cadherin expression levels in parental TFK-1 and CCLP-1 cells. Data represent the mean \pm standard deviation of at least three independent experiments $\left({ }^{*} \mathrm{P}<0.05\right)$.

A

TFK-1
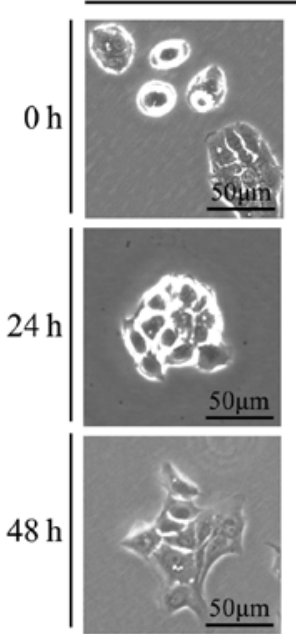

Control
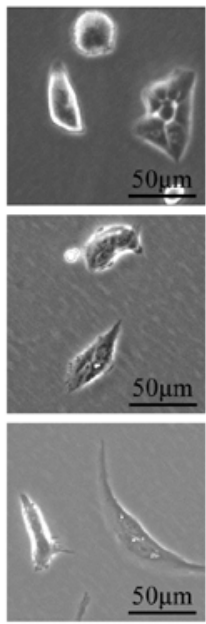

$+\mathrm{TGF}-\beta$

$(5 \mathrm{ng} / \mathrm{ml})$
B

$\mathrm{MPC1}$

E-cadherin
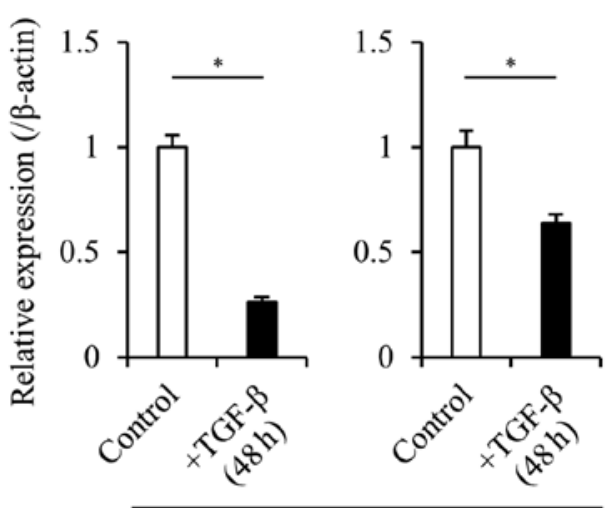

TFK-1
C

E-cadherin

MPC1

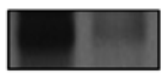

$\beta$-actin
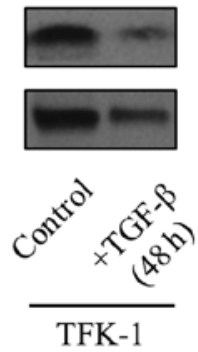

Figure 3. MPC1 expression is downregulated in cells after EMT induction by TGF- $\beta$. (A) Morphological changes observed in TFK- 1 cells treated with TGF- $\beta$ (scale bar, $50 \mu \mathrm{m}$ ). (B) Real-time qRT-PCR and (C) western blot results of MPC1 and E-cadherin expression levels in TFK-1 cells treated with or without TGF- $\beta$. Data represent the mean \pm standard deviation of at least three independent experiments $(* \mathrm{P}<0.05)$.

the proliferation assay (Fig. 5B). A wound healing assay was performed to assess changes in the cell migration in response to MPC1 gene overexpression. MPC1-overexpressing-CCLP-1 cells had a significantly lower migration ability than the parental CCLP-1 cells (Fig. 5C), which was consistent with the IHC results in which low MPC1 expression was related to both invasion and metastasis.

\section{Discussion}

We investigated the impact of the MPC1 expression on malignant potential in ICC. The IHC results revealed that low MPC1 expression correlated with poor RFS in ICC specimens and demonstrated that MPC1 expression is related to invasion and metastasis after EMT induction. We subsequently performed an in vitro study to confirm the role of MPC1 in EMT. This study revealed that MPC1 expression changed depending on the EMT state of the cancer cells and was downregulated when EMT was induced. This is the first study to assess the relationship between EMT and MPC1 expression in cancer cells.

A previous study reported that MPC1 is downregulated in various types of solid cancer (16). Consistent with the results of this study, low expression of MPC1 in prostate cancer has been revealed to correlate with poor prognosis and overexpression of MPC1 resulted in reduced invasion and cell growth in vitro (17). It is well known that cancer cells acquire their invasive and metastatic abilities through the process of EMT. Various signaling pathways, such as TGF- $\beta$, EGF and WNT/Notch are known to contribute to EMT induction (20). Recently, some studies have indicated that the molecules 
A

TFK-1

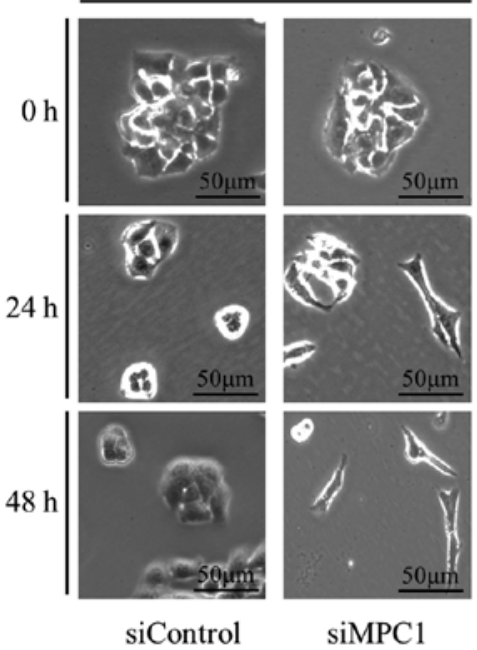

D

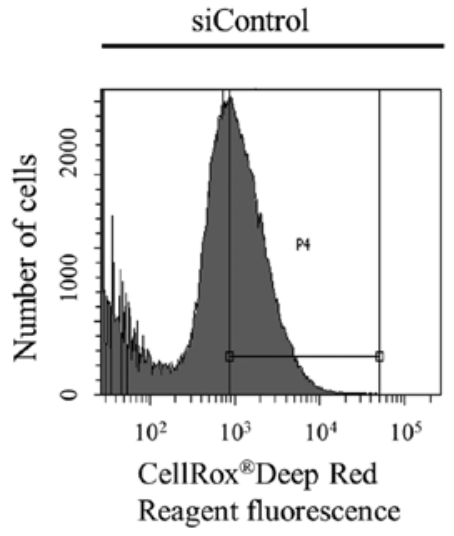

B

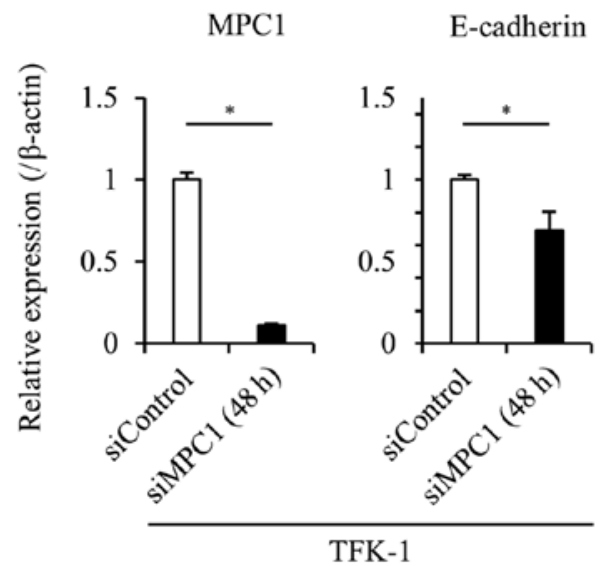

TFK-1
C

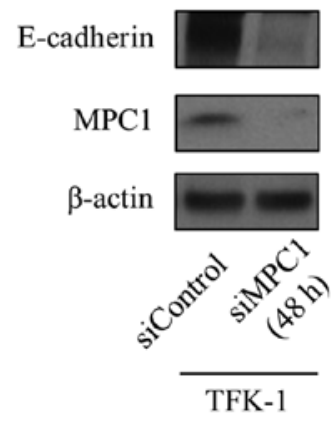

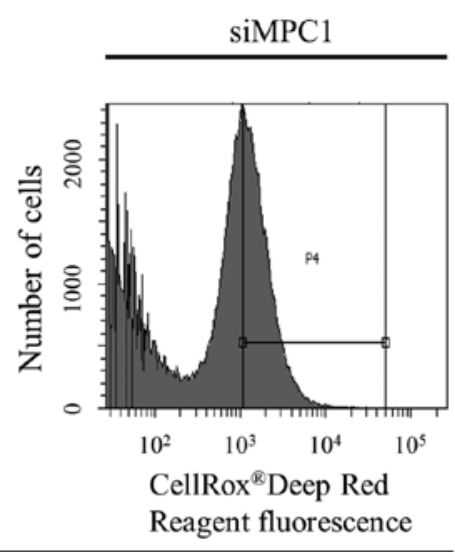

TFK-1

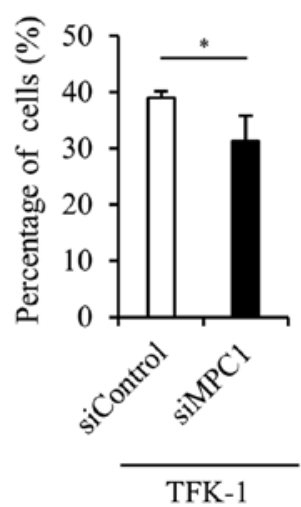

Figure 4. Knockdown of MPC1 leads to EMT induction. (A) Morphological changes in TFK-1 cells after MPC1 knockdown by siRNA transfection (scale bar, $50 \mu \mathrm{m}$ ). (B) Real-time qRT-PCR and (C) western blot results of MPC1 and E-cadherin expression levels in TFK-1 cells after MPC1 knockdown by siRNA transfection. (D) Left panels: Changes in ROS levels after MPC1 gene knockdown (left panel, control; middle panel, MPC1 gene knockdown). Right graph: Percentage of cells stained with the ROS indicator dye CellROX Deep Red. Data represent the mean \pm standard deviation of at least three independent experiments $(" \mathrm{P}<0.05)$.

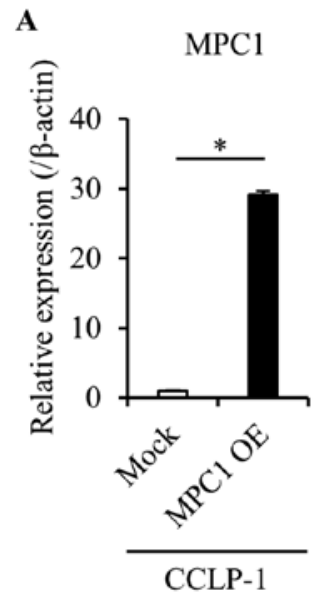

B

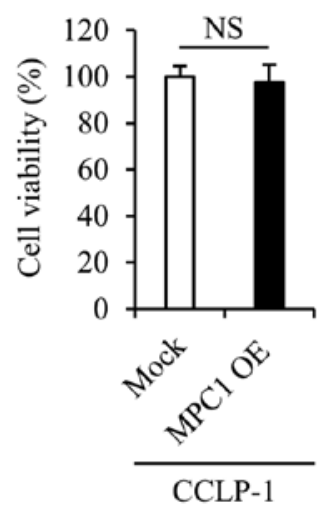

C



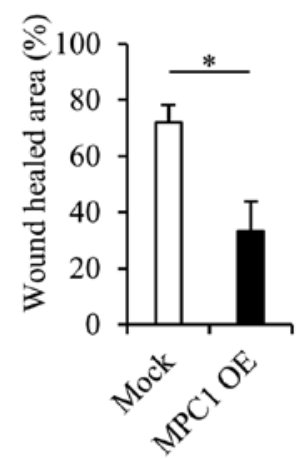

Figure 5. Tumor cell migration is suppressed by MPC1 overexpression. (A) Real-time qRT-PCR results of the MPC1 expression levels in CCLP-1 cells after transient transfection of MPC1 plasmid DNA. (B) Proliferation assay results of viable CCLP-1 cells with or without MPC1 overexpression. (C) Left: Wound healing assay results of the CCLP-1 cells with or without MPC1 overexpression. Right graph: Percentage of cells that infiltrated the wounded area. Data represent the mean \pm standard deviation of at least three independent experiments $\left({ }^{*} \mathrm{P}<0.05\right)$. 
involved in cancer metabolism are also involved in EMT induction. For instance, PKM2, an alternatively spliced variant of the pyruvate kinase gene, plays a role in EMT induction via an interaction with the transcription factor TGF- $\beta$-induced factor homeobox 2 (11). Although the detailed mechanism by which MPC1 regulates EMT induction is unclear, we hypothesized that changes in ROS levels possibly via MPC1 regulation may be involved. Given that the EMT phenomenon is associated with the production of ROS, the present study indicated that the inhibition of MPC1 stimulated the antioxidant pathway in the pentose phosphate pathway (PPP), which quenched the deleterious ROS and enhanced the EMT phenomenon of the living cells. In addition, a previous study revealed that the epigenetic silencing of the gluconeogenic enzyme fructose-1, 6-biphosphatase by the EMT-associated factor Snail decreased ROS levels (21), which could promote EMT. We confirmed that ROS levels were decreased in response to the MPC1 knockdown. The detailed mechanism of EMT induction should be further examined to better understand the function of MPC1.

Although the study of crystal structure indicates the similarity of MPC1 and MPC2 $(12,13)$, it remains to be elucidated whether homodimer or heterodimer is dominant in cancer cells. The present study indicated that MPC1 is associated with the survival of cancer patients and the malignant behaviors of cancer cells. We focused on the function of MPC1. We concluded that MPC1, but not MPC2, is associated with the histopathological features of cancer patients and with the mitochondrial metabolism in cancer cells.

In conclusion, we found that MPC1 functions as a modulator of EMT induction and contributes to the malignant potential in ICC. As MPC1 downregulation is observed in a wide variety of solid cancers, these findings indicate that MPC1 may serve as a novel therapeutic target, not only in ICC but also in other types of cancer.

\section{Acknowledgements}

We wish to thank the members of our laboratories for their fruitful discussions. In addition, this study was supported in part by a Grant-in-Aid for Scientific Research from the Ministry of Education, Culture, Sports, Science, and Technology (K.O., Y.D., M.M., and H.I.); a Grant-in-Aid from the Ministry of Health, Labor and Welfare (K.O., Y.D., M.M., and H.I.); a grant from P-DIRECT (H.I.); a grant from the National Institute of Biomedical Innovation (M.M. and H.I.); and a grant from Osaka University Drug Discovery Funds (M.M. and H.I.).

\section{References}

1. Kobayashi S, Igami T, Ebata T, Yokoyama Y, Sugawara G, Mizuno T, Nimura $Y$ and Nagino M: Long-term survival following extended hepatectomy with concomitant resection of all major hepatic veins for intrahepatic cholangiocarcinoma: Report of a case. Surg Today 45: 1058-1063, 2015.
2. Mizuno T, Ebata T, Yokoyama Y, Igami T, Sugawara G, Yamaguchi $J$ and Nagino M: Adjuvant gemcitabine monotherapy for resectable perihilar cholangiocarcinoma with lymph node involvement: A propensity score matching analysis. Surg Today 47: 182-192, 2017.

3. Wellner UF, Shen Y, Keck T, Jin W and Xu Z: The survival outcome and prognostic factors for distal cholangiocarcinoma following surgical resection: A meta-analysis for the 5-year survival. Surg Today 47: 271-279, 2017.

4. Ebata T, Ercolani G, Alvaro D, Ribero D, Di Tommaso L and Valle JW: Current status on cholangiocarcinoma and gallbladder cancer. Liver Cancer 6: 59-65, 2016.

5. Kroemer G and Pouyssegur J: Tumor cell metabolism: Cancer's Achilles' heel. Cancer Cell 13: 472-482, 2008.

6. Pavlova NN and Thompson CB: The emerging hallmarks of cancer metabolism. Cell Metab 23: 27-47, 2016.

7. Zhao Y, Butler EB and Tan M: Targeting cellular metabolism to improve cancer therapeutics. Cell Death Dis 4: e532, 2013.

8. Liberti MV and Locasale JW: The Warburg Effect: How does it benefit cancer cells? Trends Biochem Sci 41: 211-218, 2016.

9. Vander Heiden MG, Cantley LC and Thompson CB: Understanding the Warburg effect: The metabolic requirements of cell proliferation. Science 324: 1029-1033, 2009.

10. Le A, Cooper CR, Gouw AM, Dinavahi R, Maitra A, Deck LM, Royer RE, Vander Jagt DL, Semenza GL and Dang CV: Inhibition of lactate dehydrogenase A induces oxidative stress and inhibits tumor progression. Proc Natl Acad Sci USA 107: 2037-2042, 2010.

11. Hamabe A, Konno M, Tanuma N, Shima H, Tsunekuni K, Kawamoto K, Nishida N, Koseki J, Mimori K, Gotoh N, et al: Role of pyruvate kinase M2 in transcriptional regulation leading to epithelial-mesenchymal transition. Proc Natl Acad Sci USA 111: 15526-15531, 2014.

12. Bricker DK, Taylor EB, Schell JC, Orsak T, Boutron A, Chen YC, Cox JE, Cardon CM, Van Vranken JG, Dephoure N, et al: A mitochondrial pyruvate carrier required for pyruvate uptake in yeast, Drosophila, and humans. Science 337: 96-100, 2012.

13. Herzig S, Raemy E, Montessuit S, Veuthey JL, Zamboni N, Westermann B, Kunji ER and Martinou JC: Identification and functional expression of the mitochondrial pyruvate carrier. Science 337: 93-96, 2012.

14. Patterson JN, Cousteils K, Lou JW, Manning Fox JE, MacDonald PE and Joseph JW: Mitochondrial metabolism of pyruvate is essential for regulating glucose-stimulated insulin secretion. J Biol Chem 289: 13335-13346, 2014.

15. Vanderperre B, Bender T, Kunji ER and Martinou JC: Mitochondrial pyruvate import and its effects on homeostasis. Curr Opin Cell Biol 33: 35-41, 2015.

16. Schell JC, Olson KA, Jiang L, Hawkins AJ, Van Vranken JG, Xie J, Egnatchik RA, Earl EG, DeBerardinis RJ and Rutter J: A role for the mitochondrial pyruvate carrier as a repressor of the Warburg effect and colon cancer cell growth. Mol Cell 56: 400-413, 2014

17. Wang L, Xu M, Qin J, Lin SC,Lee HJ, Tsai SY and Tsai MJ: MPC1, a key gene in cancer metabolism, is regulated by COUPTFII in human prostate cancer. Oncotarget 7: 14673-14683, 2016.

18. Li X, Ji Y, Han G, Li X, Fan Z, Li Y, Zhong Y, Cao J, Zhao J, Zhang M, et al: MPC1 and MPC2 expressions are associated with favorable clinical outcomes in prostate cancer. BMC Cancer 16: 894, 2016

19. Zhao QJ, Yu YB, Zuo XL, Dong YY and Li YQ: Milk fat globule-epidermal growth factor 8 is decreased in intestinal epithelium of ulcerative colitis patients and thereby causes increased apoptosis and impaired wound healing. Mol Med 18: 497-506, 2012.

20. Xu J, Lamouille S and Derynck R: TGF-beta-induced epithelial to mesenchymal transition. Cell Res 19: 156-172, 2009.

21. Dong C, Yuan T, Wu Y, Wang Y, Fan TW, Miriyala S, Lin Y, Yao J, Shi J, Kang T, et al: Loss of FBP1 by Snail-mediated repression provides metabolic advantages in basal-like breast cancer. Cancer Cell 23: 316-331, 2013. 